Instituto Internacional de Investigación y Desarrollo Tecnológico Educativo INDTEC, C.A.

DOI: https://doi.org/10.29394/scientific.issn.2542-2987.2017.2.4.7.124-136

OAI-PMH: http://www.indteca.com/ojs/index.php/Revista Scientific/oai

\title{
Estrategias Didácticas que Fomenten el Cuidado y Conservación del Agua
}

\author{
Autora: Enma Rosa Montilla Rangel \\ Universidad Pedagógica Experimental Libertador, UPEL \\ montillaenmaem@gmail.com \\ Barinas, Venezuela
}

\section{Resumen}

La presente investigación tiene como objetivo fundamental desarrollar estrategias didácticas que fomenten el cuidado y conservación del agua en el CEIB Barinitas. Parroquia Barinitas, Municipio Bolívar del estado Barinas. La naturaleza de la investigación estuvo enmarcada bajo el paradigma cualitativo y el tipo de investigación es la investigación acción, bajo un diseño de campo con un apoyo descriptivo cumpliendo las cuatro (4) fases planteadas por Murcia (1994), (Diagnostico, Planificación, Ejecución y Evaluación). La técnica aplicada para recabar la información fue la entrevista y la observación participante. De la misma manera, se utilizó como instrumento un guion de entrevista. Asimismo, para dar respuestas a las preguntas formuladas en el guion de entrevista se tomaron como informantes claves a tres (3) docentes pertenecientes a la institución en estudio. Los criterios de calidad de la investigación se basaron en fiabilidad, credibilidad, sistematicidad, confianza, transferibilidad y la confirmabilidad del instrumento. Para llevar a cabo el análisis e interpretación de la información se realizó a través del proceso de triangulación de datos. Los resultados obtenidos arrojaron la necesidad de ejecutar un plan acción para el fomento, cuidado y preservación del agua en los niños y niñas del Centro de Educación Inicial Bolivariano, Barinitas. Como conclusión se evidencio la intensión de los docentes de participar en jornadas de capacitación para mejorar las estrategias didácticas que fomenten el cuidado y preservación del agua en los educandos. y se recomendó a los directivos y coordinadores pedagógicos llevar un seguimiento y control para que las estrategias sean incorporadas a las clases diarias.

Palabras clave: estrategias didácticas; agua; cuidado y preservación. 


\title{
Didactic Strategiesthat Foster Care and Water Conservation
}

\begin{abstract}
The present research has as main objective to develop didactic strategies that promote the care and conservation of water in the CEIB Barinitas. Barinitas Parish, Barinas state Bolivar County. The nature of the research was framed under the qualitative paradigm and the type of research is action research, under a field design with a descriptive support fulfilling four (4) phases raised by Murcia (diagnosis, planning, implementation and evaluation). The technique used to collect data was the interview and participant observation. In the same way, it was used as an instrument an interview script. Also, to respond to questions in the interview guide were taken as key informants to three (3) teachers from the institution under study. The quality criteria of the investigation were based on reliability, credibility, systematic, trust, transferability and Confirmability the instrument. To carry out the analysis and interpretation of the information was made through the process of data triangulation. The results obtained have shown the need to run an action plan for the promotion, care and preservation of water in children Early Education Center Bolivarian, Barinitas. In conclusion the intention of teachers to participate in training sessions to improve teaching strategies that promote the care and preservation of water in the students was evident. and the directors and pedagogical coordinators was recommended to track and control strategies are incorporated into daily lessons.
\end{abstract}

Keywords: teaching strategies; water; care and preservation. 


\section{Introducción}

El agua es un recurso esencial para la humanidad y uno de los principales elementos de la vida de todos los seres vivos. A lo largo de la historia el agua ha condicionado la vida de las comunidades y ha sido un factor clave en el desarrollo económico, social, industrial y humano de los pueblos. Además, la misma constituye más del cincuenta por ciento $(50 \%)$ del peso corporal de los seres vivos y es el principal nutriente y oxigenante que ellos poseen. Al respecto, Raymond (2010) dice que:

El agua es el componente principal de la materia viva. Constituye del 50 al $90 \%$ de la masa de los organismos vivos. El protoplasma, que es la materia básica de las células vivas, consiste en una disolución de grasas, carbohidratos, proteínas, sales y otros compuestos químicos similares en agua (pág. 54).

En efecto, el agua es uno de los elementos más importantes de la naturaleza, se obtiene fundamentalmente por su caída a la tierra en forma de lluvia; fluye por la superficie terrestre creando arroyos, ríos y lagunas, está en constante movimiento de acuerdo a la topografía por donde se desliza. De esta manera, el agua viaja siguiendo la trayectoria que le marcan los suelos, los declives, las quebradas y hondonadas, formando lo que se llaman cuencas, de donde se extrae para el consumo humano.

En Venezuela la situación de escasez del agua ha llegado al punto de convertirse en una prioridad, los venezolanos han tenido que utilizarla exclusivamente para usos necesarios como aseo personal y del hogar, y muchas veces no les alcanza para cubrir todas las necesidades. Así lo plantean Aguilera y Azocar (2003) cuando señalan que:

En Venezuela existen zonas críticas donde están disminuyendo los recursos hídricos, como consecuencia, por una parte del aumento de los centros poblados y áreas de desarrollos agropecuarios e industriales, y por otra, de la mejor calidad del agua exigida para el uso potable industrial y de cría (pág. 112) 
De hecho, algunas regiones de ese país han venido teniendo un crecimiento demográfico muy descontrolado; otras que están practicando el desarrollo agropecuario con productos químicos y están presentando una disminución acelerada del recurso hídrico; Entre ellos, el agua potable, causando grandes problemas sociales ya que las personas se están viendo en la obligación de darle un uso muy racional y aun así no les da vasto para cubrir sus necesidades.

En estado Barinas, según fuentes de la empresa Estatal Hidroandes (2013), "las principales fuentes y caudales de agua dulce de donde se extrae el vital líquido a través de acueducto están en peligro de desaparición" (pág. 13). Por lo planteado, es necesario que se tomen las medidas pertinentes en cuanto a la recuperación de las nacientes de agua y de las cabeceras de los ríos que presentan graves problemas de deterioro, mediante la conservación, protección, mitigación, compensación, uso racional y aprovechamiento sostenible de estas. Al respecto Cañizares (2006) señala que "el agua es un factor indispensable para el desarrollo y su presencia se traduce en mejor calidad de vida, no habiendo desarrollo sustentable sin su existencia, pues todos los actos humanos están relacionados con ella" (pág. 202). En este sentido, es necesario concienciar la población ante su despilfarro y mal uso que se le da a este vital e indispensable líquido, para generar un uso racional otorgando la posibilidad de encontrar una mejor calidad de vida.

A esta situación no escapa el centro de Educación Inicial bolivariano, Barinitas, el cual ha venido presentando constantes problemas en cuanto al uso racional y consciente del agua por parte de los estudiantes que hacen vida en la institución; los cuales al momento de dirigirse al baño hacer sus necesidades fisiológicas o ha cepillarse los dientes, sin el acompañamiento de las docentes; los niños y niñas dejan los grifos de los baños abiertos, provocando grandes pérdidas de volumen de agua potable, desatendiendo las 
problemáticas de escasez de agua que ha venido presentando el Municipio, siendo el agua un elemento indispensable para el desarrollo de la vida.

\section{Estrategias.}

En toda acción educativa para el desarrollo cognitivo de los educandos, los profesores tienen que hacer uso de las estrategias y si verdaderamente se quiere que los niños(as) desarrollen sus habilidades, destrezas, técnicas, el docente debe seleccionar con mucha responsabilidad la estrategia adecuada que permita en el menor tiempo, y con el menor esfuerzo alcanzar los objetivos previstos, por ello es fundamental que el educador sea un experto en la aplicación de las estrategias.

En este orden de ideas, las estrategias para Serna (2001: 35), son las "acciones que deben realizarse para mantener y soportar el logro de los objetivos de la organización y de cada unidad de trabajo, y así, hacer realidad los resultados esperados al definir los proyectos estratégicos". Las estrategias son aplicables en las diferentes instituciones donde existe una planificación para lograr un fin, cumplir una meta, obtener resultados y alcanzar objetivos propuestos.

\subsection{Estrategias Didácticas}

La estrategia didáctica, es el conjunto de procedimientos que, apoyados en técnicas de enseñanza, tienen por objeto llevar a buen término la acción didáctica. Para mayor comprensión del contenido, iniciaremos con la definición del concepto, desde la perspectiva de diversos autores. G. Avanzini (1998). Considera que las estrategias didácticas requieren de la correlación y conjunción de tres componentes: misión, estructura curricular y posibilidades cognitivas del alumno. 


\subsection{Intencionalidad Didáctica de las Estrategias.}

La intención didáctica de una estrategia es la búsqueda de hacer de manera más fácil el proceso enseñanza aprendizaje al educando cuyo propósito es enriquecer la evolución del sujeto. Al respecto, Rangel, (2010), la define como el arte de saber transmitir los conocimientos de la forma más adecuada para su asimilación. En tal sentido, El currículo escolar es un sistema de vertebración institucional de los procesos de enseñanza y aprendizaje, y tiene fundamentalmente cuatro elementos constitutivos: objetivos, contenidos, metodología y evaluación.

\subsection{Tipos de Estrategias Didácticas.}

Díaz y Hernández ubican los diferentes tipos de estrategias en tres grandes grupos a los que definen del siguiente modo:

2.3.1. Estrategias de apoyo: se ubican en el plano afectivo-motivacional y permiten al aprendiz mantener un estado propicio para el aprendizaje. Pueden optimizar la concentración, reducir la ansiedad ante situaciones de aprendizaje y evaluación, dirigir la atención, organizar las actividades y tiempo de estudio, etcétera.

2.3.2. Estrategias de aprendizaje 0 inducidas: procedimientos $y$ habilidades que el alumno posee y emplea en forma flexible para aprender y recordar la información, afectando los procesos de adquisición, almacenamiento y utilización de la información.

2.3.3. Estrategias de enseñanza: consisten en realizar manipulaciones o modificaciones en el contenido o estructura de los materiales de aprendizaje, o por extensión dentro de un curso o una clase, con el objeto de facilitar el aprendizaje y comprensión de los alumnos. Son planeadas por el agente de enseñanza (docente, diseñador de materiales o software educativo) y deben utilizarse en forma inteligente y creativa (1). 


\subsection{El agua}

El término agua generalmente se refiere a la sustancia en su estado líquido, aunque la misma puede hallarse en su forma sólida llamada hielo, y en su forma gaseosa denominada vapor. Tal como lo define (Ruiz, 2001):

El agua es un elemento de gran abundancia en el planeta, y es un componente muy importante de todos los seres vivos. Es una fuerza moldeadora de paisajes y aun mas, es un factor clave en la climatización del globo, que permite la existencia de la vida y el desarrollo de los pueblos. (pág. 11).

De la misma manera, es definida por Isturiz (1992), como:

El agua a causa de deficiencias en su manejo y aprovechamiento, se ha convertido en un problema social, tanto por su aparente abundancia, que eventualmente provoca catástrofes, como por cierta escasez estacional o regional, o por tener una calidad inadecuada para el uso humano debido a su estado natural (pág. 61).

Lo planteado por los autores determina que el agua es un componente indispensable para el desarrollo y la supervivencia de los seres vivos. Además, es un elemento clave en el desarrollo de los pueblos, por lo que su preservación y el buen uso son imprescindibles en la existencia del globo terráqueo.

\subsection{Estructura y propiedades del agua}

La molécula de agua está formada por dos átomos de $\mathrm{H}$ unidos a un átomo de $\mathrm{O}$ por medio de dos enlaces covalentes. El ángulo entre los enlaces $\mathrm{H}-\mathrm{O}-\mathrm{H}$ es de 104' 5․ El oxígeno es más electronegativo que el hidrógeno y atrae con más fuerza a los electrones de cada enlace.

El resultado es que la molécula de agua, aunque tiene una carga total neutra (igual número de protones que de electrones), presenta una distribución asimétrica de sus electrones, lo que la convierte en una molécula polar, 
alrededor del oxígeno se concentra una densidad de carga negativa, mientras que los núcleos de hidrógeno quedan parcialmente desprovistos de sus electrones y manifiestan, por tanto, una densidad de carga positiva.

\subsection{Recomendaciones Sobre el Consumo de Agua}

Como norma general, debemos beber en los intervalos entre comidas, entre dos horas después de comer y media hora antes de la siguiente comida. Está especialmente recomendado beber uno o dos vasos de agua nada más levantarse. Así conseguimos una mejor hidratación y activamos los mecanismos de limpieza del organismo.

\section{Metodología}

La presente investigación está enmarcada bajo el paradigma cualitativo y el estudio está enfocado en la investigación de campo. Tal como lo señala el Manual de Trabajos de Grado de Maestría de la Universidad Experimental Libertador (UPEL, 2006). "es la que analiza sistemáticamente los problemas de la realidad, con la intención de detallarlos, explicarlos, concebir su naturaleza y los componentes que explican sus causas y consecuencias". De acuerdo a lo planteado, se propone un plan estrategias didácticas que fomenten el cuidado y preservación del agua para niños y niñas del Centro de Educación Inicial Bolivariano, Barinitas, de la Parroquia Barinitas, Municipio Bolívar del Estado Barinas.

De igual forma, la investigación se tipifica como descriptiva, por cuanto en la misma se describe de forma real la situación presentada. Al respecto, Hurtado (2008: 108) señalan que en la investigación descriptiva se: "trata de obtener información acerca del estado actual de los fenómenos. Con ellos se logra precisar la naturaleza de la situación, tal como existe en el momento de estudio". 
De la misma manera, el diseño del presente proyecto corresponde con la metodología de investigación acción la cual es definida por Martínez (2000) como aquella que reúne un conjunto de elementos permitiendo guiar el estudio en términos practico-operativo y donde los resultados se describen usando el lenguaje del discurso cotidiano empleado por los participantes" (pág. 131).

Elliot, (1991), por su parte, considera la investigación-acción como:

El estudio de una situación social para intentar mejorar la calidad de la acción en la misma. Su objetivo consiste en proporcionar elementos que sirvan para facilitar el juicio práctico en situaciones concretas y la validez de las teorías e hipótesis que genera no depende tanto de pruebas 'científicas' de verdad, sino de su utilidad para ayudar a las personas a actuar de modo más inteligente y acertado. En la investigación acción, las 'teorías' no se validan de forma independiente para aplicarlas luego a la práctica, sino a través de la práctica (pág. 88).

Según lo planteado por los autores, considera que la investigación de acción, es adecuada para el presente estudio porque es un proceso de continua búsqueda, entiende que la interacción humana y la intervención social no pueden ser tratados como procesos mecánicos, sino como procesos permanentes de construcción colectiva que genera cambio social y conocimiento educativo sobre la realidad social o educativa, proporcionando entre otras ventajas y autonomía.

\section{Descripción de la Metodología}

Fase I. Diagnóstico: origina el primer momento de búsqueda, de indagación, acompañado de un gran deseo de comprender el por qué ocurren las cosas. La reflexión en el momento del diagnóstico permite descubrir las debilidades tanto de los alumnos como del propio docente y tomar conciencia de las estrategias implementadas que no le han dado resultado, por lo que requieren ser cambiadas. Permite conocer los problemas o situaciones que 
afectan a un grupo o comunidad. Por ello una vez identificado el significado del problema, y, formulado el enunciado del mismo, se debe proceder.

Fase II. Planificación: analizada e interpretada la información, se deriva a pensar y visualizar algunas alternativas de actuación, que permitirán diseñar una propuesta de cambio, cuyas actividades darán forma y estructura a la investigación. Esta planificación debe considerar objetivos a lograr, recursos necesarios, lapsos de tiempo en los cuales se ejecutará, responsable de cada acción. De igual manera, resulta pertinente, definir su evaluación, es decir, anticipar los indicadores que darán cuenta de su logro. De allí, que en el presente estudio, el plan de acción partirá de las iniciativas y consensos logrados durante las interacciones con los involucrados en la investigación.

Fase III Ejecución: una vez diseñada la planificación, esta se lleva a cabo mediante la acción grupal; las cuales pueden dejar significativas experiencias acerca de lo que se ventila en el estudio. A propósito de esta etapa de ejecución, Murcia (1990), señala que es "la fase de la puesta en práctica de los planes acordados" (pág. 20). Agrega además, que del enfrentamiento con el o los problemas, "permiten el conocimiento más profundo de la realidad, pues éste se logra cuando se intenta transformarla" (ídem).

Fase IV Evaluación: esta etapa se presenta como posterior a la de ejecución, pero generalmente, esto no es así, porque precisamente, al final durante el desarrollo de cada jornada es necesario obtener información acerca de los logros alcanzados y de las experiencias adquiridas en función de los objetivos pretendidos. Es decir, la evaluación, además de ser aplicada en cada momento, debe estar presente al final de cada ciclo, dando de esta manera una retroalimentación a todo el proceso.

Asimismo, la técnica empleada en esta investigación es la entrevista estructurada, son aquellas en que el entrevistador se vale de una guía de preguntas específicas y se sujeta exclusivamente a estas. Según Buendía, 
Colás y Hernández (2009), es: "la recogida de información a través de un proceso de comunicación, en el transcurso del cual el entrevistado responde a cuestiones previamente diseñadas en función de las dimensiones que se pretenden estudiar planteadas por el entrevistador y las mismas se dividen en estructuradas, semiestructuradas o no estructuradas o abiertas" (pág. 83).

En este sentido, las entrevistas estructuradas, son aquellas en que el entrevistador se vale de una guía de preguntas específicas y se sujeta exclusivamente a estas.

En este orden de ideas, los informantes claves, son las personas o sujetos que darán respuesta a la entrevista, aportando opiniones relacionadas con las categorías y subcategorías necesarias para llegar a la teorización y comprensión del tema en estudio. Según Márquez (1994) "son un grupo de personas con conocimientos especiales, estatutos y buena capacidad de información. Un buen informante clave puede desempeñar un papel decisivo y trascendental en una investigación" (pág. 199). Es por ello que, en todo el proceso se busca establecer una relación de confianza con los informantes a objeto de recabar la información necesaria y de interés para la investigación. Para la realización de la presente investigación, la población quedará conformada por los tres (3) docentes de aula del CEIB Barinitas, de la parroquia Barinitas, Municipio Bolívar, Estado Barinas.

Igualmente, como técnica de análisis de los resultados se realizó a través de la categorización; siendo esta, una forma de clasificación de la que deriva otras unidades más pequeñas llamadas subcategorías. Esta agrupa elementos y características derivados de la categoría en estudio.

\section{Consideraciones Finales}

De acuerdo a los objetivos planteados en la presente investigación se llegaron a las siguientes consideraciones: 
Se evidencio que los docentes del Centro de Educación inicial Bolivariano Barinitas, utilizan pocas estrategias que ayuden al fomento del cuidado y preservación del agua en los niños y niñas de la institución educativa.

De la misma manera, se evidencio la intensión de los docentes de participar en jornadas de capacitación para mejorar las estrategias didácticas que fomenten el cuidado y preservación del agua en los educandos

Asimismo, se recomendó a los directivos y coordinadores pedagógicos llevar un seguimiento y control para que las estrategias sean incorporadas a las clases diarias.

\section{Referencias}

Aguilera y Azocar. (2003). Ciencias e Hidrografía. Instituto venezolano de investigaciones científicas. Primera Edicion. Caracas. CAPATE. Cañizares, C. (2006). El ambiente. Segunda Edición. CAPATE.

Hurtado, R. (2008). Metodología de la Investigación. Editorial Episteme. Caracas - Venezuela.

Martínez, M. (2000). La investigación cualitativa etnográfica en educación. 3ra Edición. Trillas

Raymond, L. (2010). El agua y la Vida. REMACA.

Serna, F. (2001). Procesos de Aprendizaje. 1ra Edición. Caracas.

Universidad Pedagógica Experimental Libertador (2006). Manual de Trabajo de grado, Especialización y Tesis Doctoral. Caracas. 


\section{Enma Rosa Montilla Rangel \\ e-mail: montillaenmaem@gmail.com}

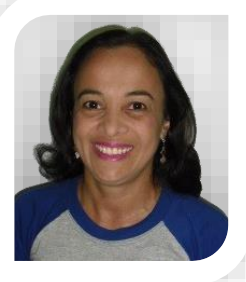

Nacida en Barinitas, Estado Barinas, Venezuela. Cursa estudio de Maestría en Educación Ambiente y Desarrollo en la Universidad Pedagógica Experimental Libertador en Barinitas Estado Barinas. Profesora en Educación Inicial en la Universidad Pedagógica Experimental Libertador (2004). Técnico Superior en Educación Preescolar en el Tecnológico Agustín Codazzi (2008).

Actualmente se desempeña como Docente de Aula en el Preescolar Bolivariano Barinitas en el Municipio Bolívar del Estado Barinas. Desde el 2006 hasta 2016. Se desempeñó durante un año de servicio como Docente de Atención no Convencional en el Preescolar Terrazas del Caipe Barinas Estado Barinas.

Asimismo, ha participado como ponente del I congreso internacional de innovación y calidad educativa organizado por la UNELLEZ en el año 2014, entre otros.

El contenido de este manuscrito se difunde bajo una Licencia de Creative Commons ReconocimientoNoComercial-Compartirlgual 4.0 Internacional 